\title{
Characterization of (357439) 2004 BL86 on its close approach to Earth in 2015
}

\author{
M. Birlan ${ }^{1}$, M. Popescu ${ }^{2,1}$, D.A. Nedelcu ${ }^{2}$, V. Turcu ${ }^{3}$, A. Pop ${ }^{3}$, B. Dumitru ${ }^{4}$, F. Stevance ${ }^{5,6}$, O. Vaduvescu ${ }^{5}$, \\ D. Moldovan ${ }^{3}$, P. Rocher ${ }^{1}$, A. Sonka ${ }^{2}$, and L. Mircea ${ }^{3}$ \\ ${ }^{1}$ Institut de Mécanique Céleste et de Calcul des Éphémérides (IMCCE), Observatoire de Paris, CNRS UMR8028, \\ 77 avenue Denfert-Rochereau, 75014 Paris Cedex, France \\ e-mail: Mirel.Birlan@imcce.fr \\ 2 Romanian Academy, Astronomical Institute, 5 Cuţitul de Argint, 75212 Bucharest, Romania \\ 3 Romanian Academy, Astronomical Institute, Astronomical Observatory Cluj, 19 Cireşilor, 400487 Cluj-Napoca, Romania \\ 4 Institute for Space Science, 409-Atomiştilor, 077125 Magurele, Ilfov, Romania \\ 5 Isaac Newton Group of Telescopes, Apartado de Correos 321, 38700 Santa Cruz de la Palma, Canary Islands, Spain \\ ${ }^{6}$ Department of Physics and Astronomy, University of Sheffield, Sheffield S3 7RH, UK
}

Received 4 May 2015 / Accepted 2 July 2015

\begin{abstract}
Context. The potentially hazardous asteroid (PHA) (357439) 2004 BL86 grazed Earth on January 26, 2015 at a distance of about 1.2 million $\mathrm{km}$. This favorable geometry allowed observing it to derive its physical and dynamical parameters. (357439) 2004 BL86 was previously estimated to be a 500-m body. We study it also considering possible mechanisms that might mitigate the effect of asteroids that might become dangerous for Earth.

Aims. Physical and dynamical investigations of this peculiar object were performed to be able to characterize this object.

Methods. We used spectral observations obtained in the visible (V) using the Isaac Newton Telescope and in the near-infrared (NIR) using the InfraRed Telescope Facility. A complementary photometric survey during two nights was also provided by the Astronomical Observatory Cluj-Feleacu station in Romania. We anlyzed the data using reliable mathematical tools that were previously published under the acronym M4AST.

Results. VNIR spectral observations classify (357439) 2004 BL86 as V-type asteroid. The mineralogical analysis reveals its similarities to howardite-eucrite-diogenite meteorites. The band analysis reveals that the object is more similar to a eucritic and howarditic composition, and that it originated from the crust of a large parent body. The analysis yields a mineralogical solution of $\mathrm{Wo}_{17} \mathrm{Fs}_{39}$ with an error bar of $4 \%$. Based on the average value of the thermal albedo for V-type objects, its diameter was re-estimated to a value of $290 \pm 30 \mathrm{~m}$. The dynamical analysis shows a chaotical behavior of (357439) 2004 BL86. The statistics on meteorite falls show that (357439) 2004 BL86 does not appear to significantly contribute to the current howardite-eucrite-diogenite meteorite flux. For the two photometrical observing runs the following values of the rotational period and peak-to-peak amplitude were estimated for the light curves: $2.637 \pm 0.024 \mathrm{~h}, 0.105 \pm 0.007 \mathrm{mag}$, and $2.616 \pm 0.061 \mathrm{~h}, 0.109 \pm 0.018 \mathrm{mag}$, respectively.
\end{abstract}

Key words. minor planets, asteroids: individual: (357439) 2004 BL86 - methods: observational - techniques: spectroscopic

\section{Introduction}

Near-Earth asteroids (NEAs) is a term that describes all small bodies that intersect the orbits of telluric planets. The updated catalog contains 12552 objects $^{1}$. Of these, 1579 objects are classified as potentially hazardous asteroids (PHAs). Of these PHAs, only 40 present spectral data covering the visible and near-infrared interval. PHAs are NEAs whose minimum orbital intersection distance (MOID) with Earth is shorter than 0.05 AU and whose absolute magnitude is smaller than 22 .

Over the geological history of our planet, collisions with asteroids have altered the course of life, and there is no reason that these unpredictable events should not continue. Detailed analyses of questions related to realistic options for preventing the collision of a NEA with Earth are now performed in Europe (Harris et al. 2013). Several similar initiatives for providing detailed

\footnotetext{
1 Number until April 30, 2015, http://neo.jpl.nasa.gov/ stats/
}

test mission designs for mitigation processes are now developed all over the world. These initiatives include both researchers in fundamental sciences and industries specialized in space explorations to establish a correct scenario of each situation. Each scenario is specific to each NEA that is subject to mitigation. Thus, a good knowledge of the physical properties of NEAs and the response of various types of external stress is required to design an efficient mitigation tool (Michel 2013).

As a result of their vicinity to Earth, PHAs contribute to a large part of the flux of particles that are trapped by the gravitational field of Earth. The mass flux of meteoroids of between $10 \mathrm{~g}$ to $1 \mathrm{~kg}$ size to Earth was estimated to between 2900 and $7300 \mathrm{~kg} / \mathrm{yr}$ (Bland et al. 1996). Several ejection mechanisms of particles from asteroids (i.e., contribution to the meteoroid flux in the vicinity of Earth) were proposed (Jewitt et al. 2015), but the efficiency of these mechanisms is still a subject of debate. Undeniably, the physics, mass distribution, morphology, and composition of asteroids are constraints for solving this problem. 
A\&A 581, A3 (2015)

Table 1. Observational circumstances during the spectroscopic observations of asteroid (357439) 2004 BL86.

\begin{tabular}{lccccccccccc}
\hline \hline Date (UT) & $\begin{array}{c}V \\
(\mathrm{mag})\end{array}$ & $\begin{array}{c}\Phi \\
(\mathrm{o})\end{array}$ & $\begin{array}{c}\Delta \\
(\mathrm{AU})\end{array}$ & $\mathrm{AM}$ & $\begin{array}{c}T_{\text {exp }} \\
(\mathrm{min})\end{array}$ & $\begin{array}{c}I_{\text {time }} \\
(\mathrm{s})\end{array}$ & $\mathrm{Cy}$ & $\begin{array}{c}S \\
\left({ }^{\prime \prime}\right)\end{array}$ & $\begin{array}{c}H \\
(\%)\end{array}$ & $\begin{array}{c}\text { Spectrum } \\
(\mu \mathrm{m})\end{array}$ & Analog \\
\hline Feb. 7, 2015, 10h 22m & 16.5 & 50.7 & 0.107 & 1.66 & 12 & $120 \mathrm{~s}$ & 3 & 0.9 & 19 & $0.8-2.5$ & HD 72911 \\
Feb. 6, 2015, 02h 30m & 16.4 & 50.6 & 0.095 & 1.30 & 40 & $600 \mathrm{~s}$ & 4 & 1.0 & 15 & $0.4-0.9$ & HD 76617 \\
\hline
\end{tabular}

References. We list the date, mid-time exposure time, apparent magnitude, phase angle, distance Earth-asteroid, airmass (AM), total exposure time $\left(T_{\text {exp }}\right)$, individual exposure time and number of cycles, seeing $(S)$, humidity $(H)$, spectral interval, and solar analogs.

While NEAs evolve on orbits much closer to Earth than main belt asteroids, the common idea might be that they can be observed more easily and more often. But their small diameters imply tight constraints that are related to their close encounters with Earth. These encounters occur on average three to five times per century (Popescu et al. 2011). It is mandatory to take advantage of these favorable geometries ${ }^{2}$ to perform as many observations as possible to constrain the mass, internal structure, surface composition and rugosity, etc. A key-point science in these studies is the characterization of objects with small diameters (smaller than $0.5 \mathrm{~km}$ ).

Asteroid (357439) 2004 BL86 grazed Earth on January 26, 2015 at a distance of about 1.2 million $\mathrm{km}$. This asteroid was discovered on 30 March 2004 in the framework of the LINEAR program (Stokes et al. 2000). Its absolute magnitude was computed to be 19.4, which allows an estimate of the diameter of between 400-550 m, assuming typical albedo values for asteroids between 0.1 and 0.2. Its osculating elements using 1004 individual observations from the Minor Planet Center database were computed for the epoch December 9, 2014, $0^{\mathrm{h}} \mathrm{TT}$ with the values $a=1.5022021 \mathrm{AU}, e=0.4030734, i=23.7436134$, $\omega=311^{\circ} .2548538, \Omega=126^{\circ} .7203833$, and $M=354^{\circ} .0330939$ using Asterpro (Rocher 2007). Asterpro is a complex tool developed by Patrick Rocher from IMCCE for adjusting observations and computing the osculating elements for asteroids, ephemerides in various references systems (apparent, geocentric, barycentric, etc), and elaborating precompiled ephemerides using Chebyshev polynomial representations. These osculating elements were then used to compute the next favorable geometry of observation for January-February 2050, when the asteroid will pass at $0.0071 \mathrm{AU}$ during its next closest approach to Earth.

There are almost no published data in the literature concerning this object. The Central Bureau of Astronomical Telegrams published the CBET number 4063, of which a short report of a coordinated campaign of photometry and radar observations reveals that the object is a binary system, with the satellite diameter approximately six to eight times smaller than that of the primary ${ }^{3}$.

To better characterize this object during its closest approach to Earth on 26th of January 2015, we obtained spectra in the visible and the near-infrared in two consecutive nights in February 2015 in the frame of regular programs. A photometric survey during two observing nights was also provided by the Astronomical Observatory Cluj-Feleacu station in Romania. We here present the results of these runs, which include light curve analysis and mineralogical modeling of the composite spectrum.

\footnotetext{
2 These geometries are usually between 5-30 days of visibility.

3 http://www. cbat.eps.harvard.edu/iau/cbet/004000/ CBET004063.txt
}

\section{Observations}

Near-infrared (NIR) spectral observations $(0.8-2.5 \mu \mathrm{m})$ were carried out using the upgraded SpeX instrument mounted on the InfraRed Telescope Facility (IRTF), located on Mauna Kea, Hawaii. The remote observing technique was used from CODAM-Paris Observatory (Birlan et al. 2004). The upgraded SpeX (uSpeX) instrument was used in low-resolution prism mode (Rayner et al. 2003), with a $0.8 \times 15^{\prime \prime}$ slit oriented northsouth. Spectra of the asteroid and solar analogs were obtained alternatively in two distinct locations on the slit (A and B); this is referred to as the nodding procedure.

The visible $(\mathrm{V})$ spectrum $(0.4-0.9 \mu \mathrm{m})$ was obtained using the IDS instrument mounted on Isaac Newton Telescope (INT), located at El Roque de Los Muchachos Observatory, Canary Islands. These observations were obtained remotely in a first remote observing run between the ROC-Astronomical Institute, Romanian Academy (Nedelcu et al. 2014; Birlan et al. 2014a) and INT. The IDS instrument was used in low-resolution mode (R150 grating) with a slit width of $1.5^{\prime \prime}$ and the RED+2 CCD detector.

For both V and NIR observations, solar analog G2V stars were scheduled to be observed in the apparent vicinity of the asteroid. Table 1 summarizes the observational circumstances of our spectroscopic observations.

NIR data reduction was carried out by means of standard procedures using the new version of the Spextool pipeline (Cushing et al. 2004) for uSpeX. The median flat-field for each night was constructed, then all images were corrected with this median flat-field. To eliminate the sky contribution, the A and B images were subtracted, and the resulting A-B were added for the whole integration time period. The final images were collapsed to a two-dimensional pixel-flux matrix. The result obtained was then calibrated in wavelength, using the argon lamp lines. The calibration was performed using the solar analog HD 72911, observed after data acquisition for our object, at similar airmass.

The visible spectrum was obtained using the new pipeline dedicated to the INT/IDS instrument. This new pipeline is as yet unpublished and was written in Octave and IRAF (Popescu et al., in prep.). The first step of the pipeline consists of bias and flatfield corrections. Then, the unidimensional spectra are automatically extracted after a visual selection of images ${ }^{4}$. Wavelength calibration is done based on the lines of a $\mathrm{CuAr}+\mathrm{CuNe}$ lamp. The result of these steps is a matrix containing the wavelength, the intensity at each wavelength, and the error for each value of intensity. Finally, the division of the asteroid spectrum with the G2V solar analog is computed. If a wavelength shift appears

\footnotetext{
4 Visual inspection of the images and manual deletetion of those that have poor $\mathrm{S} / \mathrm{N}$ or are contaminated by a star should precede this operation.
} 
between the asteroid and the solar analog spectra (the so-called heartbeats), the routines also reduce its influence onto the relative reflectance. In this case, the analog HD 76617 was used to obtain the relative reflectance of the asteroid.

Photometric monitoring was made at the Astronomical Observatory Cluj - Feleacu Station in Romania. The equipment used for the observations was a Meade LX200 16-inch telescope equipped with a CCD SBIG camera STL 6303E used in $2 \times 2$ analog binning mode, with a FOV of $23.7^{\prime} \times 15.8^{\prime}$. Two photometric time series were obtained for the night of 31 st of January 2015 and 3rd of February 2015, using the Cousins I filter. (357439) 2004 BL86 was observed for more than five hours (spanning the phase angle interval $47.98^{\circ}-48.28^{\circ}$ ) in good photometric conditions on the night of 31 st of January 2015. The integration time for each image was 25 seconds, and the median seeing was stable throughout the sequence, to the value $1.56^{\prime \prime}$. SAO 15258 was used as comparison star, along with the following check stars: GSC 4148:1187, GSC 4148:464, and GSC 4148:664. The role of check stars is to verify the stability in magnitude of the comparison star over the run. During the night 3rd of February 2015, the photometric sequence covered more than four hours (the corresponding phase angle interval was $50.24^{\circ}-50.30^{\circ}$ ). The photometric conditions were good, and the median seeing was 1.26". Because of the significant increase in distance of the asteroid - Earth compared with the first observing night, the integration time for each image was set to $40 \mathrm{~s}$. The comparison star and the check stars used were GSC 4151:671, GSC 4151:461, GSC 4151:432, GSC 4151:373, and GSC 4151:388, respectively.

The image series calibration procedure (bias, dark, flatfield, defect map) and aperture photometrical reduction for ClujFeleacu were performed with the AIP4Win V2.10 software (Berry \& Burnell 2005).

\section{Results}

\subsection{Photometry}

Photometric data were modeled using a mathematical model consisting of the superposition of a truncated Fourier series and a low-order polynomial trend (Pop et al. 2004 and references herein). The values of coefficients were estimated through nonlinear least-squares fitting, where the input value of the variation frequency was determined from the analysis of the light-curve amplitude spectrum of the detrended light curve. The light-curve profile is shown in Figs. 1 and 2. The mathematical formalism is presented in the Appendix.

The amplitude spectrum of the detrended data obtained during the night between January 31 and February 1, 2015 contains the peaks corresponding to the fundamental (rotation) frequency together with its four harmonics (Fig. A.1). The value of the fundamental frequency is $8.7 \mathrm{cy} /$ day, while the respective peak is the highest in the spectrum. It is interesting that the amplitude spectrum of the detrended data obtained during the second observing night, February 3/4, 2015 (Fig. A.2) only contains three peaks associated with the asteroid rotation: the fundamental (rotation) frequency ( $8.2 \mathrm{cy} /$ day) and its two harmonics, their peaks having larger amplitudes. The corresponding improved rotational frequency values are $9.103 \pm 0.084$ and $9.18 \pm 0.21 \mathrm{cy} /$ day, respectively. This evolution of the amplitude spectrum structure can be understood by taking into account the interplay between the surface inhomogeneities and the change of the orientation of the asteroid rotation axis with respect to the line of sight (Kaasalainen et al. 2001). This explanation agrees with the change in the shape

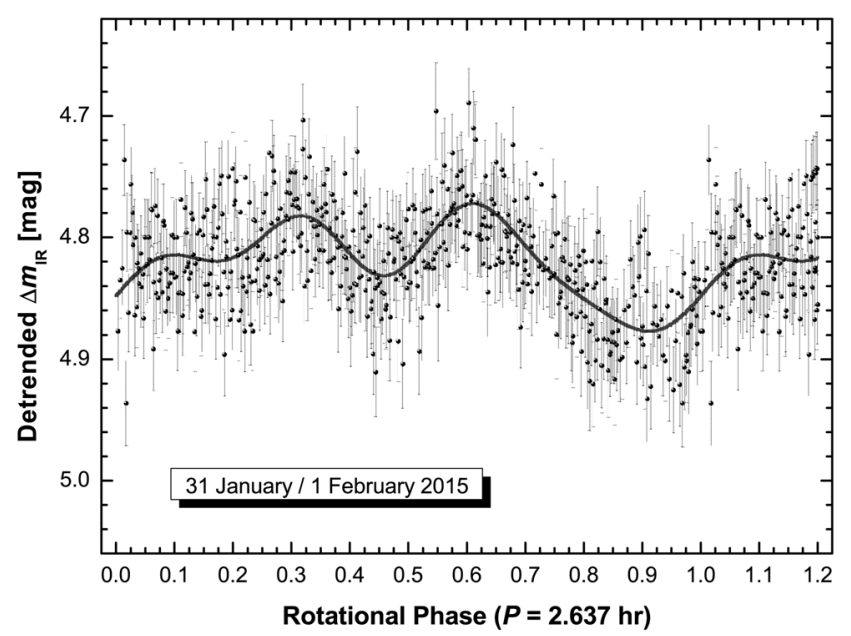

Fig. 1. Light curve of 2004 BL86 for the night of January 31-February $1,2015$.

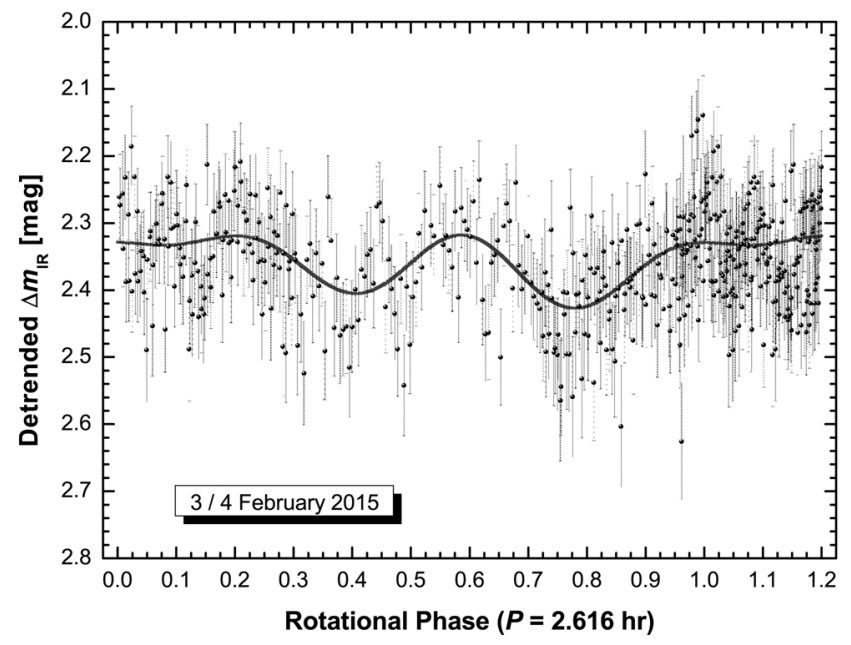

Fig. 2. Light curve of 2004 BL86 for the night of February 3/4, 2015.

of the light curve between the two observing runs, as can be seen in Figs. 1 and 2. For the two nights, the following values of the rotational period and peak-to-peak amplitude were estimated for the light curves: $2.637 \pm 0.024 \mathrm{~h}, 0.105 \pm 0.007 \mathrm{mag}$, and $2.616 \pm 0.061 \mathrm{~h}, 0.109 \pm 0.018 \mathrm{mag}$, respectively.

These values are in relatively good agreement with those stored by NEODyS-2 ${ }^{5}$, and published by CBET number 4063 .

\subsection{Spectroscopy}

The omposite VNIR spectrum of (357439) 2004 BL86 is presented in Fig 3. The VNIR spectrum was obtained by merging the visible part with the infrared one for the common interval 0.78-0.94 $\mu \mathrm{m}$ (Popescu et al. 2012). The composite spectrum was normalized to unity at $0.55 \mu \mathrm{m}$. Figure 3 shows that the differences in the common wavelength interval between the visible and the near-infrared part are smaller than the error bars of the two spectra. This is expected since the aspect of the object does not change very much between the moments of V and NIR observations. The match also confirms the correctness of the two data reduction procedures.

\footnotetext{
http://newton.dm. unipi .it/neodys/index $\cdot$ php?pc=1 1 . $9 \& n=357439$
} 


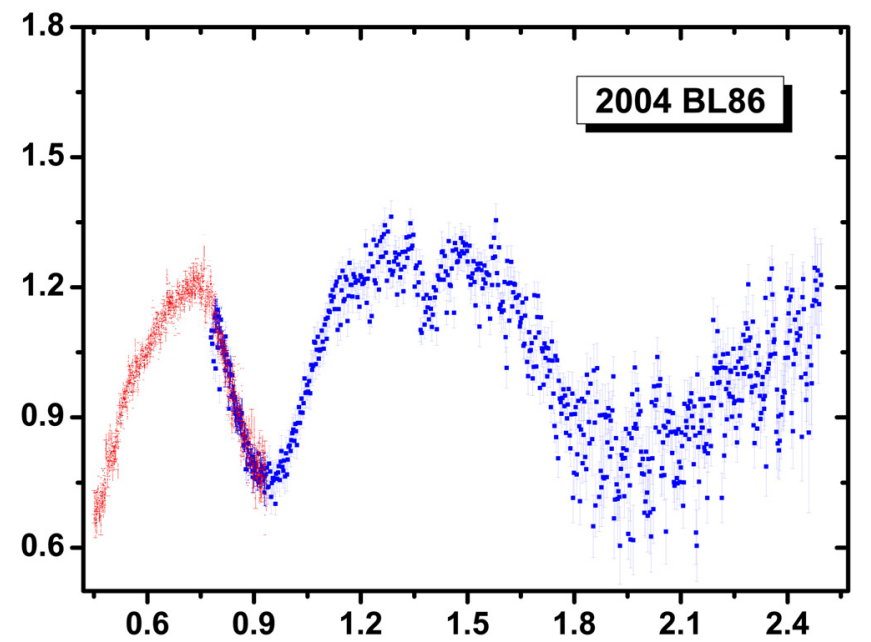

Fig. 3. Composite VNIR spectrum of (357439) 2004 BL86 normalized to unity at $0.55 \mu \mathrm{m}$.

If we consider a thermal albedo of V-type objects estimated from NEOWISE data around 0.362 \pm 0.035 (Mainzer et al. 2011) or even an older estimate from IRAS data $(0.422 \pm 0.084$ in Fulchignoni et al. 2000), using an absolute magnitude of 19.4 we can derive a diameter in the range of $290 \pm 30 \mathrm{~m}$, approximately $40 \%$ smaller that the one computed previously. Our computed diameter value agrees with the one informally reported by radar ${ }^{6}$ or by other spectral measurements ${ }^{7}$.

Two prominent absorption features in the reflectance spectrum of 2004 BL86 occur near $1 \mu \mathrm{m}$ and $2 \mu \mathrm{m}$ and are associated with the presence of olivine $(\mathrm{Ol})$ and pyroxene $(\mathrm{Px})$ mineralogies. While pyroxene exhibits the two absorption bands, olivine is only characterized by a complex broad absorption band around $1 \mu \mathrm{m}$.

The VNIR spectrum of 2004 BL86 was modeled using the procedures of the M4AST tool, which are described in detail by Popescu et al. (2012). The analysis performed is similar to that used for the asteroid (809) Lundia (Birlan et al. 2014b).

A fist step into our analysis is to classify the asteroid using the Bus-DeMeo taxonomy (DeMeo et al. 2009). The M4AST tool (Popescu et al. 2012) classifies (357439) 2004 BL86 as a V-type object. The same result was obtained using the SMASS-MIT tool ${ }^{8}$.

We analyzed our spectrum using the model of Cloutis et al. (1986) that was updated by Dunn et al. (2010) after applying the temperature correction of the band centers (Burbine et al. 2009). Band $I$ and $I I$ minima were computed as $0.9506 \pm 0.0014 \mu \mathrm{m}$ and $1.9508 \pm 0.0204 \mu \mathrm{m}$, respectively. The band area ratio (BAR) was estimated to be $1.860 \pm 0.006$. These values place the object in the region corresponding to the basaltic achondrite minerals (band I-BAR diagram of Gaffey et al. 1993). The estimate of the relative proportion of olivine to ortho-pyroxene was computed using the BAR value and Eq. (4) of Gaffey et al. (2002). Using the results of Dunn et al. (2010), we computed an $\mathrm{OPx} /(\mathrm{OPx}+\mathrm{Ol})$ value of 0.827 . This is a strong indicator that the surface is mainly covered by ortho-pyroxene. When the band $I$ and $I I$ minima are placed in the context of pyroxene types of

\footnotetext{
6 CBET number 4063 and mpml web list, http://www.jpl.nasa. gov $/$ news . php? feature $=4459$

7 http://www.psi.edu/news/ceresopnav2

8 http://smass.mit.edu/busdemeoclass.html
}

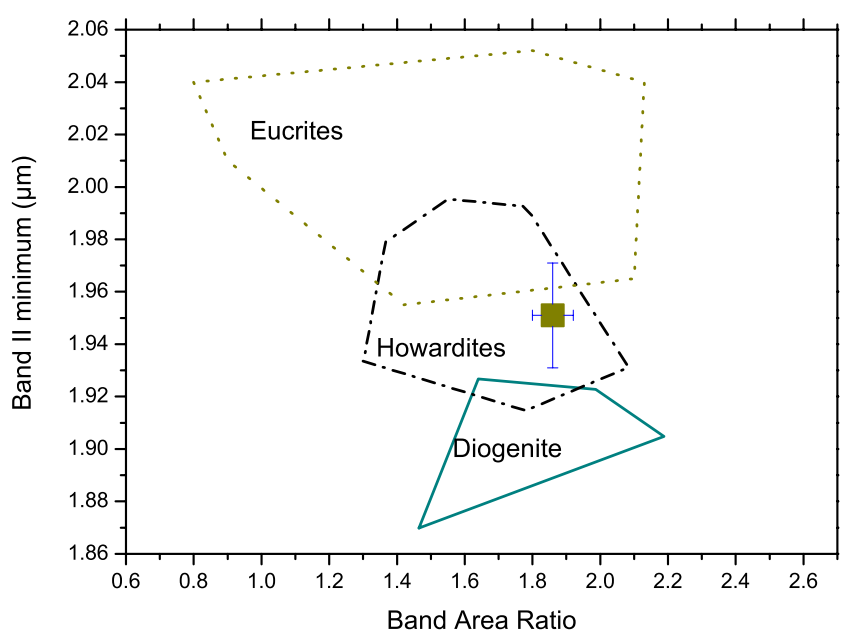

Fig. 4. Mineralogical parameters for the HED diagram of Moskovitz et al. (2010). The composition is more like eucritic and howarditic mineralogy.

crystals, that is, ortho-pyroxene (OPx) and clino-pyroxene (CPx) (Adams 1974), we come to similar results.

The spectral characteristics of 2004 BL86 were also computed using the empiric model proposed by Gaffey et al. (2002) that was refined by Burbine et al. (2009). This iterative procedure allows estimating the pyroxene composition in terms of molar $\mathrm{Ca}$ and $\mathrm{Fe}$ content. These computations converge toward a low-calcium content, typical of orthopyroxenes, the solution being $\mathrm{Wo}_{17} \mathrm{Fs}_{39}$ with an error of $4 \%$.

The band separation was used to estimate the iron content (de Sanctis et al. 2011) of the mineralogical matrix. Laboratory calibration indicates that the band separation given as a function of the band II minimum shows a linear trend that can be correlated with the iron content (Cloutis et al. 1990). The measurements match this linear trend quite well, and we computed the iron content and obtained $35 \pm 2 \%$.

Specific areas for howarditic, eucritic, and diogenitic meteorites in the space BAR-band II center were published by Moskovitz et al. (2010). The values computed for (357439) 2004 BL86 show a composition of the object that is more similar to eucritic and howarditic mineralogy. These measurements placed our object in the region where these two mineralogies overlap (Fig. 4). This indicates that 2004 BL86 is most probably a crustal piece of a large V-type parent body. Given that all the NEAs with MOIDs smaller than 0.1 AU are potential sources of meteorites (Olsson-Steel 1988), (357439) 2004 BL86 might contribute to the current howardite-eucrite-diogenite (HED) meteorite falls.

Comparative mineralogy was performed using laboratory spectra from the Relab database ${ }^{9}$ by means of M4AST routines (Popescu et al. 2012). Different spectral matching methods were used to find the best fit with meteorite spectra. These methods include least-quares errors, $\chi^{2}$, and the correlation coefficient between the asteroid spectrum and those of meteorites.

Overall, the first 50 spectral analogs found by the procedure belong to eucritic and howarditic meteorites. For most of the best matches, the grain sizes are relatively large. These matches support the hypothesis that strong rugosity may be present on its surface. Table 2 lists the five best matches for our composite spectrum.

9 http://www.planetary . brown.edu/relabdocs/relab.htm 
Table 2. Results obtained by matching spectra of (357439) 2004 BL86 with laboratory spectra from the Relab database.

\begin{tabular}{lllll}
\hline \hline Asteroid & Meteorite & Sample ID & Type & Size $(\mu \mathrm{m})$ \\
\hline \multirow{2}{*}{ 357439) 2004 BL86 } & EETA79005 & TB-RPB-026 & HED eucrite polymict & $0-250$ \\
& EETA79006,66 & MP-TXH-123 & HED eucrite polymict & $0-125$ \\
& EET83251 & TB-RPB-022 & HED eucrite polymict & $0-1000$ \\
& Petersburg & MR-MJG-097 & HED howardite & unknown \\
& PCA82501,24 & MP-TXH-124 & HED eucrite unbrecciated & $0-125$ \\
\hline
\end{tabular}

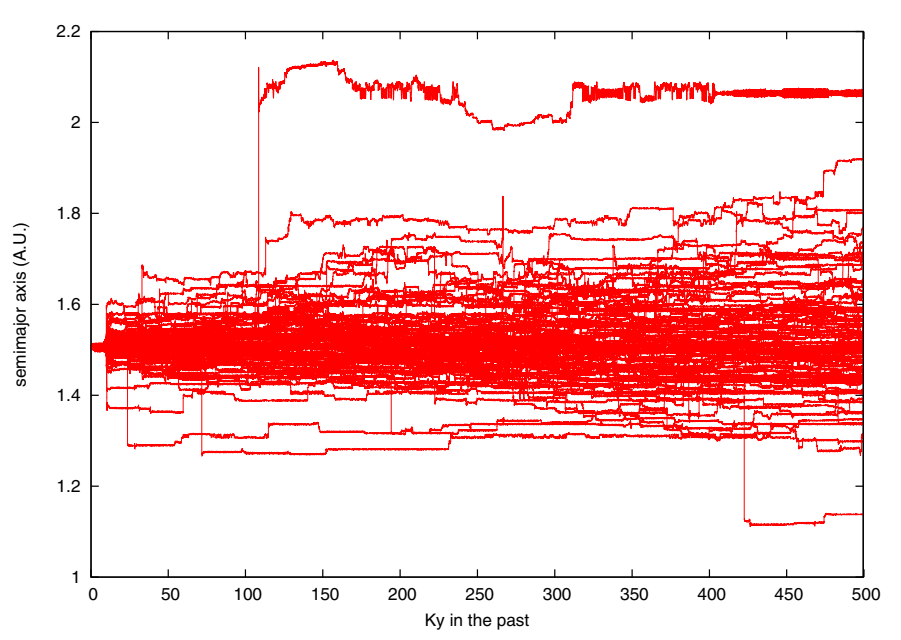

Fig. 5. Evolution of the semi-major axis for 100 clones integrated backward in time for a period of 500000 years.

\subsection{Dynamics}

We investigate the orbital evolution of (357439) 2004 BL86 backward in time using a statistics based on 100 clones well described in (Nedelcu et al. 2014).

The past orbital evolution of 2004 BL86 analyzed by a numerical integration of 100 clones displays a markedly chaotic behavior. As a result of close planetary approaches, the initial population strongly diverges after only 8000 years, with individual clones being captured for short periods in the resonances between 1.2 and 1.8 AU. A single clone moved to $q<0.05 \mathrm{AU}$ region and was removed from the population. The other objects remained in the NEA space for the entire 500000-year period (Fig. 5).

\section{Discussion}

A sample of V-type objects exists also among NEAs. There are very few objects among PHAs that are assigned to a V-type taxonomic class. (357439) 2004 BL86 together with other V-type asteroids (DeMeo et al. 2009; Bus \& Binzel 2002; Sanchez et al. 2013; Binzel et al. 2004) are representative for this taxonomic class within PHAs and are probably related to HED meteorites.

2004 BL86 was reported as a binary asteroid by radar measurements. Together with asteroid 2006 BT18 (Reddy et al. 2008), these are the two known objects among PHAs that belong to the V-type taxonomic class.

However, dynamically NEAs objects at the origin of HED meteorites were not yet identified precisely. HED meteorites are mainly of basaltic structure. While spectra similar to basalt were identified in the main belt, we can speculate on the origin of basaltic NEAs and their reservoir inside the main belt. The main reservoir associated with basaltic asteroids is the associated with the family of asteroid (4) Vesta (Binzel \& Xu 1993) or other differentiated asteroids that are completely destroyed during the time of the dynamically excited asteroid belt (Lazzaro et al. 2000; Hardersen et al. 2004; Duffard 2009). The remnant chips of differentiated objects are injected by mechanisms of drift and resonance pumping to orbits crossing the orbits of Earth and Mars. As a direct consequence, the mineralogy of differentiated NEAs belonging to V-type taxonomic class is similar to the mineralogy of objects located in the main belt of asteroids.

The ratio OPx/(OPx+Ol) computed for (357439) 2004 BL86 agrees with results and statistical results obtained by spectroscopic surveys of Vesta-family members (Duffard et al. 2004). The result of forsteritic composition also agrees with the composition of the average of Vesta-family asteroids (Duffard et al. 2004). Band I and II values for 2004 BL86 are slightly shifted toward shorter wavelengths than those of other groundbased observations (Gaffey 1997). These results are consistent with the one obtained by Dawn mission (De Sanctis et al. 2013), however, and are consistent with a eucritic-howarditic mineralogy.

Another aspect that might be a subject of debate is the affinity of its spectrum to HED meteorite spectra sampled for large grain-sized particles to simulate the regolith. To avoid the inconsistency of a bias related to the RELAB database, we made an inventory of all HED spectra contained in the version of RELAB implemented for M4AST. The 174 HED individual spectra represent regolith sizes starting from $25 \mu \mathrm{m}$ until the bulk spectra of a meteorite, thus most likely the fit of the spectrum for 2004 BL86 with the large grain-sized HEDs is not affected by a database bias.

We also investigated the relation between 2004 BL86 and the HED meteorite falls. In the catalog of meteorites there is a flag that can classify them into two categories: found meteorites and fall meteorites. The category of falling meteorites includes meteorites that were found just after a fireball hit Earth, thus recording the precise period of the year of meteorite falls.

Using the Catalogue of Meteorites (Grady 2000), we have selected all the confirmed HED falls for which at least the month of fall is known (54 objects). Their distribution per month is presented in Fig. 6. The fall distribution shows periods of intense activity superimposed on a constant background flux.

The marked peak of March-April may be associated with two known V-type PHAs: 1981 Midas (MOID = 0.004 AU on March 18) and 1997 GL3 (MOID $=0.002$ AU on April 5) (Binzel et al. 2004).

For 2004 BL86, Earth approached its orbit at a distance of $0.008 \mathrm{AU}$ in January 26. Although it has a similar diameter 


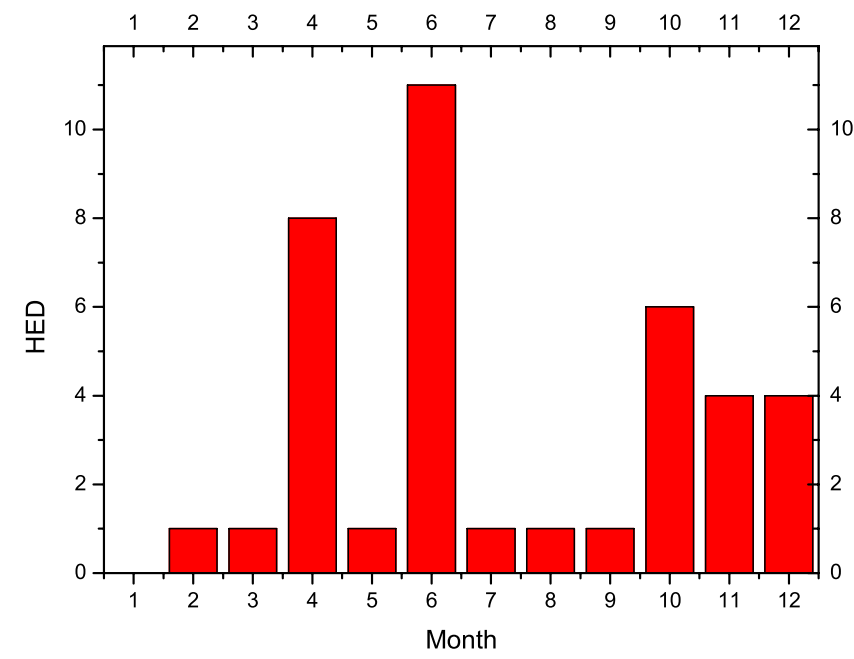

Fig. 6. Cumulative number of HEDs falls per month.

as 1997 GL3 (0.3 km in diameter), 2004 BL86 does not appear to significantly contribute to the current HED meteorites flux, as indicated by the absence of a January peak in the Fig. 6. This finding may indicate a monolith structure of the primary component of 2004 BL86, as inferred from the relatively short rotation period. The diameters of most asteroids rotating faster than $2.2 \mathrm{~h}$ are smaller than $200 \mathrm{~m}$ (Pravec \& Harris 2000).

\section{Conclusions}

Spectral VNIR and photometry of asteroid binary asteroid (357439) 2004 BL86 was obtained during its closest approach to Earth in January-February 2015.

(357439) 2004 BL86 was classified as V-type asteroid, which are particularly rare among binary PHAs. Based on an average value of the thermal albedo for a V-type object, its diameter was estimated to be $290 \pm 30 \mathrm{~m}$. The mineralogical analysis revealed similarities to HED meteorites. The band analysis revealed that the object is more similar to an eucritic and howarditic composition and that it originated from the crust of a large parent body. The analysis concludes to a mineralogical solution $\mathrm{Wo}_{17} \mathrm{Fs}_{39}$ with an errorbar of $4 \%$. The dynamical analysis showed a chaotic behavior of (357439) 2004 BL86. The result of integrating backward in time for $500000 \mathrm{yr}$ showed that this object was part of the NEA population. However, even if its MOID is $0.007 \mathrm{AU}$, we did not find a direct correlation with HED meteorite falls.

The rotational period of the asteroid was estimated to be $2.637 \pm 0.024 \mathrm{~h}$ and $2.616 \pm 0.061 \mathrm{~h}$, respectively.

The next favorable geometry for ground-based observations of (357439) 2004 BL86 will occur in January-February 2050.

Acknowledgements. The article is based on observations acquired with IRTF and INT and the CODAM and ROC remote facilities. The INT is operated on the island of La Palma by the Isaac Newton Group in the Spanish Observatorio del Roque de los Muchachos of the Instituto de Astrofisica de Canarias. This research utilizes spectra acquired by Hiroi, Pieters, Burbine, Binzel, and Gaffey, with the NASA RELAB facility at Brown University. This work was supported by a grant of the Romanian National Authority for Scientific Research Program for Research - Space Technology and Avanced Research - STAR, project number 67 and the Post-Doctoral Programme POSDRU/159/1.5/S/137516, "PARTING" project co-founded from European Social Fund through the Human Resources Sectorial Operational Program 2007-2013 and by PICS-PASSO program. AIRA ROC was funded by CNCS-UEFISCDI PN-II-RU-TE-2011-30163. This work is in support of the NEOShield-2 European Commission program.

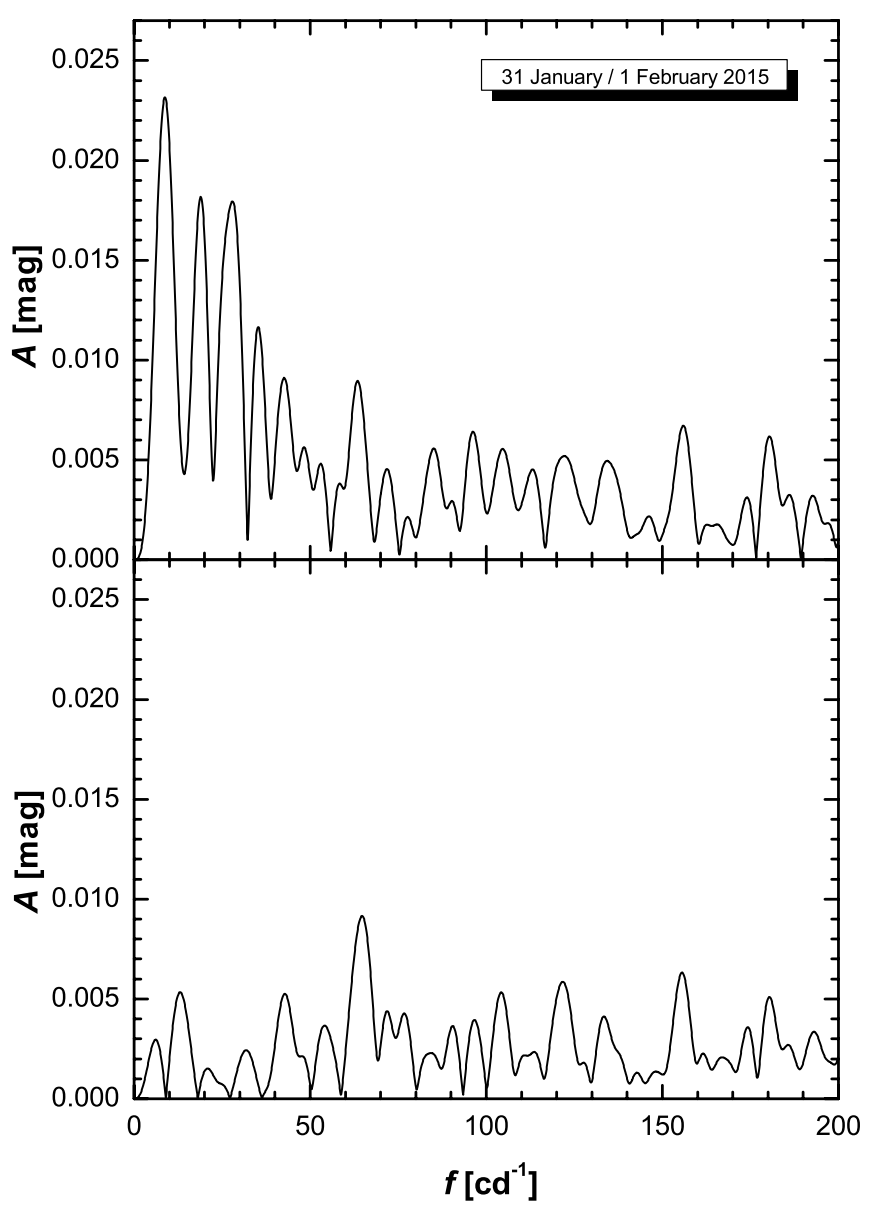

Fig. A.1. Amplitude spectrum of the detrended light curve observed during January 31-February 1, 2015 (upper panel) and the amplitude spectrum of the residuals after removing the parabolic trend and the periodic component (lower panel).

\section{Appendix A: Light curve analysis}

Each of the photometric data sets obtained during the two observing runs contains a long-term trend superposed on the variability due to the axial rotation of the asteroid. First of all, this trend was modeled with a low-order polynomial. Then, the amplitude spectrum of the residuals obtained after removing the polynomial trend was calculated to identify the frequency that corresponds to the rotational period. Finally, the asteroid light curve was described using a truncated Fourier series superposed on a low-order polynomial trend $m\left(t_{j}\right)=\sum_{k=0}^{K} m_{k} \cdot t_{j}^{k}+\sum_{l=1}^{L} A_{l} \cdot \cos \left(2 \pi l t_{j}+\Phi_{l}\right), j=1,2, \ldots, N$,

in which $m_{k}(k=0,1, \ldots, K)$ are the polynomial coefficients, $K$ is the polynomial order, $A_{l}$ and $\Phi_{l}(l=0,1,2, \ldots, L)$ are the amplitudes and the phases of the periodic terms, respectively, $L$ is the number of periodic terms, and $f$ the involved frequency, while $t_{j}(j=0,1, \ldots, N)$ are the observing times, and $N$ is the number of individual observations. The values of the model parameters were estimated through nonlinear least-squares fitting. The number of harmonics $(L-1)$ of the fundamental frequency was established according to their signature in the amplitude spectrum and their statistical significance as resulted from the modeling procedure 
M. Birlan et al.: Characterization of (357439) 2004 BL86

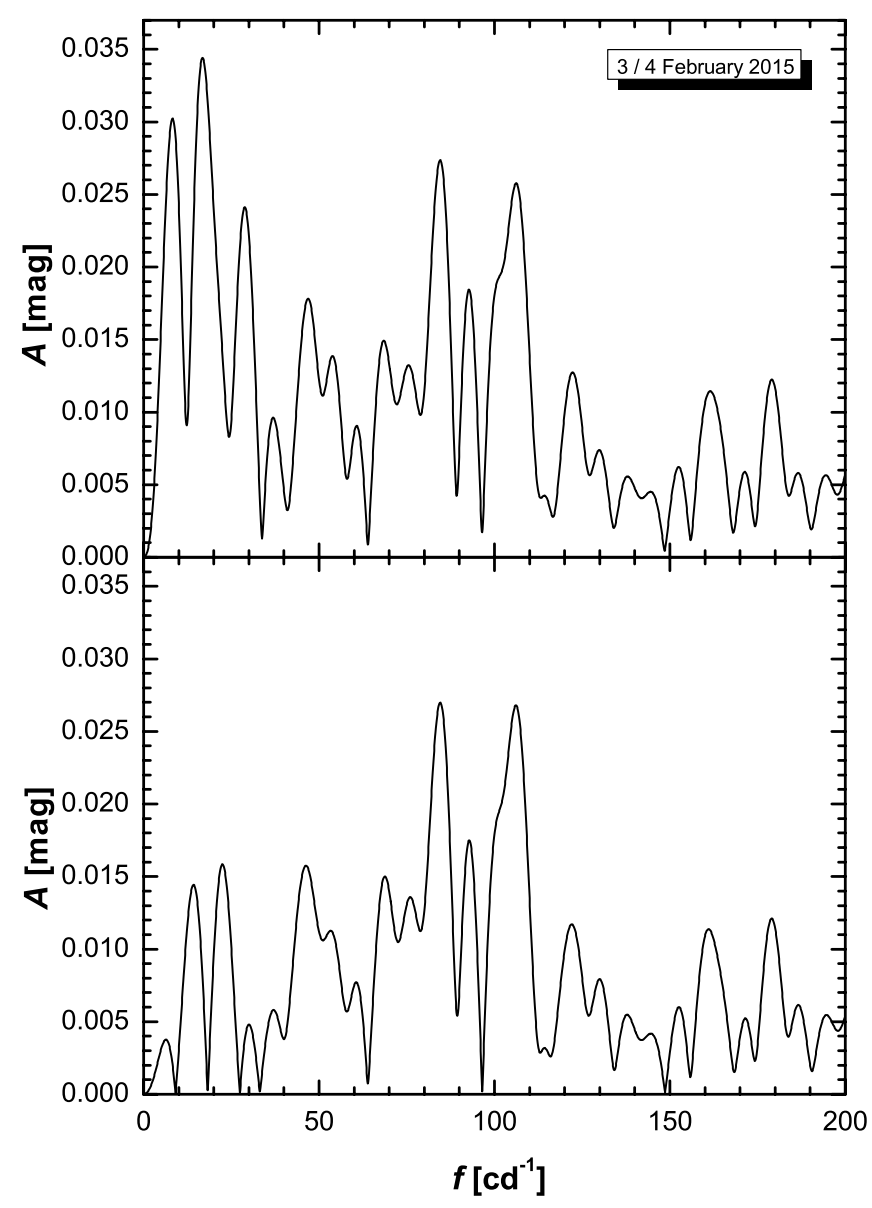

Fig. A.2. Amplitude spectrum of the detrended light curve observed during February 3/4, 2015 (upper panel) and the amplitude spectrum of the residuals after removing the parabolic trend and the periodic component (lower panel).

Table A.1. Details of photometrical observing runs, order of the polynomial trend, and the one of truncated Fourier series, together with the values of the rotational period and the peak-to-peak amplitude.

\begin{tabular}{lccccc}
\hline \hline Runs in 2015 & $N$ & $K$ & $L$ & $\begin{array}{c}\text { Rotational } \\
\text { period (h) }\end{array}$ & $\begin{array}{c}\text { Light curve } \\
\text { amplitude (mag) }\end{array}$ \\
\hline Jan. 31-Feb. 1 & 522 & 2 & 4 & $2.637 \pm 0.024$ & $0.105 \pm 0.008$ \\
Feb. 3/4 & 305 & 1 & 3 & $2.616 \pm 0.061$ & $0.109 \pm 0.018$ \\
\hline
\end{tabular}

(Pop et al. (2004), Lopez de Coca et al. (1984) and references herein). The main results of our analyses are displayed in Figs. 1, 2, A.1 and A.2 and Table A.1.

\section{References}

Adams, J. B. 1974, J. Geophys. Res., 79, 4829

Berry, R., \& Burnell, J. 2005, The Handbook of Astronomical Image Processing, 2nd. (Richmond, VA: Willmann-Bell, Inc.)

Binzel, R. P., \& Xu, S. 1993, Science, 260, 186

Binzel, R. P., Rivkin, A. S., Stuart, J. S., et al. 2004, Icarus, 170, 259

Birlan, M., Barucci, M. A., Vernazza, P., et al. 2004, New Astron., 9, 343

Birlan, M., Alin Nedelcu, D., Popescu, M., \& Badescu, O. 2014a, Romanian Astronomical Journal, 24, 119

Birlan, M., Nedelcu, D. A., Popescu, M., et al. 2014b, MNRAS, 437, 176

Bland, P. A., Smith, T. B., Jull, A. J. T., et al. 1996, MNRAS, 283, 551

Burbine, T. H., Buchanan, P. C., Dolkar, T., \& Binzel, R. P. 2009, Meteor. Planet. Sci., 44, 1331

Bus, S. J., \& Binzel, R. P. 2002, Icarus, 158, 146

Cloutis, E. A., Gaffey, M. J., Jackowski, T. L., \& Reed, K. L. 1986, J. Geophys. Res., 91, 11641

Cloutis, E. A., Gaffey, M. J., Smith, D. G. W., \& Lambert, R. S. J. 1990 J. Geophys. Res., 95, 8323

Cushing, M. C., Vacca, W. D., \& Rayner, J. T. 2004, PASP, 116, 362

de Sanctis, M. C., Migliorini, A., Luzia Jasmin, F., et al. 2011, A\&A, 533, A77

De Sanctis, M. C., Ammannito, E., Capria, M. T., et al. 2013, Meteor. Planet. Sci., 48, 2166

DeMeo, F. E., Binzel, R. P., Slivan, S. M., \& Bus, S. J. 2009, Icarus, 202, 160

Duffard, R. 2009, Earth Moon Planets, 105, 221

Duffard, R., Lazzaro, D., Licandro, J., et al. 2004, Icarus, 171, 120

Dunn, T. L., McCoy, T. J., Sunshine, J. M., \& McSween, H. Y. 2010, Icarus, 208 789

Fulchignoni, M., Birlan, M., \& Barucci, M. 2000, Icarus, 146, 204

Gaffey, M. J. 1997, Icarus, 127, 130

Gaffey, M. J., Burbine, T. H., Piatek, J. L., et al. 1993, Icarus, 106, 573

Gaffey, M. J., Cloutis, E. A., Kelley, M. S., \& Reed, K. L. 2002, Asteroids III, (Tucson: University of Arizona Press), 183

Grady, M. M. 2000, Catalogue of Meteorites (Cambridge, UK: Cambridge University Press), 696

Hardersen, P. S., Gaffey, M. J., \& Abell, P. A. 2004, Icarus, 167, 170

Harris, A. W., Barucci, M. A., Cano, J. L., et al. 2013, Acta Astronaut., 90, 80

Jewitt, D., Hsieh, H., \& Agarwal, J. 2015, in Asteroids IV, University of Arizona Space Science Series, eds. P. Michel, F. DeMeo, \& W. Bottke, in press [arXiv: 1502.02361$]$

Kaasalainen, M., Torppa, J., \& Muinonen, K. 2001, Icarus, 153, 37

Lazzaro, D., Michtchenko, T., Carvano, J. M., et al. 2000, Science, 288, 2033

Lopez de Coca, P., Garrido, R., \& Rolland, A. 1984, A\&AS, 58, 441

Mainzer, A., Grav, T., Bauer, J., et al. 2011, ApJ, 743, 156

Michel, P. 2013, Acta Astronaut., 90, 6

Moskovitz, N. A., Willman, M., Burbine, T. H., Binzel, R. P., \& Bus, S. J. 2010, Icarus, 208, 773

Nedelcu, D. A., Birlan, M., Popescu, M., Bădescu, O., \& Pricopi, D. 2014, A\&A, 567, L7

Olsson-Steel, D. 1988, Icarus, 75, 64

Pop, A., Turcu, V., \& Codreanu, S. 2004, Ap\&SS, 293, 393

Popescu, M., Birlan, M., Binzel, R., et al. 2011, A\&A, 535, A15

Popescu, M., Birlan, M., \& Nedelcu, D. A. 2012, A\&A, 544, A130

Pravec, P., \& Harris, A. W. 2000, Icarus, 148, 12

Rayner, J. T., Toomey, D. W., Onaka, P. M., et al. 2003, PASP, 115, 362

Reddy, V., Emery, J. P., \& Gaffey, M. J. 2008, BAAS, 40, 433

Rocher, P. 2007, in Notes scientifiques et techniques de l'IMCCE

Sanchez, J. A., Michelsen, R., Reddy, V., \& Nathues, A. 2013, Icarus, 225, 131

Stokes, G. H., Evans, J. B., Viggh, H. E. M., Shelly, F. C., \& Pearce, E. C. 2000, Icarus, 148, 21 